\title{
Gauge-Mediated Supersymmetry Breaking within the Dynamical Messenger Sector*
}

\author{
Erich Poppitz ${ }^{\mathrm{a}}{ }^{\dagger}$ and Sandip P. Trivedi ${ }^{\mathrm{b}}$ \\ ${ }^{a}$ Enrico Fermi Institute, University of Chicago, 5640 S. Ellis Ave., Chicago IL 60657, USA \\ ${ }^{\mathrm{b}}$ Fermi National Accelerator Laboratory, P.O. Box 500, Batavia IL 60510, USA
}

We consider the idea of combining the supersymmetry breaking and messenger sectors in models of gaugemediated supersymmetry breaking. We discuss the advantages and problems of such models, and present an existence proof by constructing an explicit example. We explore in some detail the low-energy supersymmetrybreaking dynamics and the generation of supersymmetry-breaking soft masses of the standard model superpartners, and discuss many features which are likely to be shared by more realistic models with dynamical messengers.

\section{Why dynamical messengers?}

The previous speakers in today's afternoon session discussed in detail the reasons for the current interest in gauge-mediated models of supersymmetry breaking. We refer the reader to the contributions of A. Nelson and C. Kolda to this volume for an exhaustive discussion and an extensive list of references.

We begin by giving a definition of what we mean by dynamical messengers. Loosely speaking, we will call the messenger quarks and leptons dynamical if they are an integral part of the supersymmetry breaking sector. In a more precise sense, we require that they carry quantum numbers under both the standard model gauge group, $G_{S M}$, and the gauge group responsible for the breaking of supersymmetry, $G_{S B}$.

\subsection{The problems one wants to solve.}

To motivate our interest in models with dynamical messengers - in the above well-defined sense - let us recall some features and problems of the original models of minimal gauge-mediation (MGM) of Dine, Nelson, and Shirman [1]:

\footnotetext{
*This contribution is based on an invited talk given by E. Poppitz at the Fifth International Conference on Supersymmetries in Physics, SUSY-97, Philadelphia, PA, May 27-31, 1997; Preprint EFI-97-32.

${ }^{\dagger}$ Present address: Department of Physics, University of California at San Diego, 9500 Gilman Drive, La Jolla, CA 92093, USA.
}

1. In the MGM models, the supersymmetry breaking sector is a "black box"-its only relevant feature is that it gives rise to both a supersymmetry preserving and a supersymmetry breaking vacuum expectation value of a standard model singlet chiral superfield - the so-called "messenger singlet". To realize this feature dynamically, the authors of [1] used a rather contrived "modular" approach, relying on multiple stages of symmetry breaking, generating thereby a perturbative hierarchy of scales in the supersymmetry breaking sector. In addition to being aesthetically unappealing (admittedly a rather subjective criterion!), the MGM models also require a significant amount of fine tuning (the most drastic example being the dimensionless Yukawa coupling in the " $3-2$ " model of supersymmetry breaking, which, for calculability, needs to be of order $10^{-7}$ ).

2. In addition to aesthetic and fine tuning problems, the models also face the issue that the supersymmetry breaking vacuum is (almost) always local (this was pointed out in refs. [2], [3]). It is a generic feature of models with messenger singlets that there is a supersymmetry preserving, deeper ground state. To illustrate this, recall that the messenger singlet superfield, $S$, couples in the 
superpotential to the messenger quarks and leptons, $Q, \bar{Q}\left(G_{S M}\right.$ indices are suppressed below):

$W=\lambda S Q \cdot \bar{Q}-F_{S}^{*} S$.

The last term in eq. 1.1 is added to represent the fact that when $\lambda=0$, the messenger singlet obtains a supersymmetry breaking ( $F$-type) expectation value. It is easy to see that when the Yukawa coupling $\lambda$ of the messenger singlet to the messenger quarks and leptons is nonvanishing, the equation of motion for $S, \partial W / \partial S=0$, always has a supersymmetry preserving solution with $\langle Q \bar{Q}\rangle \neq 0$ along the $D$-flat directions of $G_{S M}$. In addition to preserving supersymmetry, this minimum also breaks color and electromagnetism.

3. Finally, in the MGM models with messenger singlets, gauge symmetries alone do not forbid direct mass terms for the messenger quark and lepton superfields. Typically, one would have to impose additional global symmetries to prevent explicit mass parameters from appearing in eq. 1.1.

Our hope is that a successful model that unifies the supersymmetry breaking and messenger sectors will be able to alleviate the problems listed above. To illustrate why we think that 1.-3. might be avoided, let us imagine that the trilinear coupling in (1.1) is replaced as follows:

$\lambda S Q \bar{Q} \rightarrow \lambda[S]_{A B} Q^{A} \cdot \bar{Q}^{B}$.

In writing eq. 1.2, we have assumed that the messenger quarks and leptons now transform also in some representation of the supersymmetry breaking gauge group $G_{S B}$ ( $A$ and $B$ denote the corresponding indices). The square bracket around the field $S$ indicates that it need not be a fundamental field: it can be a product of fields (a composite) with the desired transformation property under $G_{S B}$. We hope that the problems listed above can be alleviated, since:

1. If the right $G_{S B}$ is found, one could avoid the "modular" structure of the MGM models and thus gain elegance, and avoid fine tuning.
2. Since the messenger fields are now part of the supersymmetry breaking dynamics, $G_{S B}$, the supersymmetry breaking minimum may be global. Recall that in MGM models, the coupling $\lambda$ of the messenger singlet to the messengers (1.1) causes the appearance of $G_{S M}$-breaking minima, since the messengers do not participate in supersymmetry breaking. In models where the messengers are an integral part of the supersymmetry breaking sector, this coupling may in fact be required in order to achieve supersymmetry breaking - it may be needed to lift some runaway directions.

3. Finally, if the messengers are in chiral representations of the supersymmetry breaking gauge group $G_{S B}$, direct mass terms for the messengers are forbidden by gauge invariance alone and there is no need to impose additional global symmetries.

\subsection{A new problem: loss of asymptotic freedom?}

The major problem that arises when one tries to unify the supersymmetry breaking and messenger dynamics was noted some time ago [4]: in the models that we wish to construct the messenger fields carry quantum numbers under both $G_{S B}$ and $G_{S M}$. Thus, $G_{S B}$ is a "flavor" symmetry of the messenger sector, which implies the existence of multiple copies of messenger quark and lepton supermultiplets. Conversely, $G_{S M}$ is a "flavor" symmetry of the supersymmetry breaking sector.

Now recall that the standard model gauge couplings are asymptotically free only if no more than 4 copies of messengers in the $\mathbf{5}+\mathbf{5}^{*}$ are added (for simplicity we list the field content in terms of $S U(5)$ representations). Thus, if we insist on preserving asymptotic freedom for the standard model, the representation of $G_{S B}$ under which the messenger quarks and leptons transform should have dimension less than or equal to 4 . This in turn implies that the supersymmetry breaking gauge group $G_{S B}$ should be rather "small", i.e. have representations of dimension $\leq 4$. We conclude that if we are to keep asymptotic freedom of $G_{S M}$, we require a "small" $G_{S B}$ (such that 
it has representations of dimension $\leq 4$ ) with a "large" enough unbroken flavor symmetry, such that $G_{S M} \subset G_{\text {flavor }}$.

Given our limited experience (since Seiberg's work in 1994) in building theories that dynamically break supersymmetry, constructing a supersymmetry breaking sector that has the properties listed above appears hard. Typically, achieving large flavor symmetries is difficult, without having to add explicit mass terms. Moreover, verifying that the flavor symmetry (part of which is identified with $G_{S M}$ ) is unbroken by the supersymmetry breaking dynamics is presently only possible in models where the infrared dynamics is calculable. We note that we have constrained our considerations to the case when the supersymmetry breaking gauge group is a simple group or, at most, $G_{S B}=G_{1} \times G_{2}$, since the dynamics of models with more than two groups in the product is usually much harder to analyze. However, as A. Nelson has informed us, it may be possible to construct a satisfactory model without Landau poles of $G_{S M}$ if $G_{S B}$ is a product of three "small" gauge groups, e.g. $G_{S B}=S U(2) \times S U(3) \times S U(4)$.

An interesting possibility, which would allow us to dispose of the requirement of asymptotic freedom, is that the Landau poles of the standard model gauge couplings may only signal the breakdown of our description at higher energies, rather than a fundamental inconsistency of the theory. As was realized by Seiberg [5], an infrared free theory can be just the effective low-energy weakly coupled description of an ultraviolet free theory, which becomes strongly coupled in the infrared. The "canonical" example is the $S U\left(N_{c}\right)$ supersymmetric QCD with $N_{c}+2 \leq N_{f} \leq 3 N_{c} / 2$. This theory is free in the ultraviolet and strongly coupled in the infrared. It has a weakly coupled description at low energies, given by an infrared free, "magnetic" $S U\left(N_{f}-N_{c}\right)$ gauge theorythis infrared free theory would be the analog of the standard model with a large number of extra vectorlike messengers. If such a scenario is realized, the true description of the theory at short distances could be quite different from the familiar $S U(3) \times S U(2) \times U(1)$. However, at this stage of our understanding it appears hard to build explicit models that realize this possibility, be- cause Seiberg's duality is not well understood at nonzero momentum and in the presence of supersymmetry breaking, and because there are no "compelling" (i.e. simple, weakly coupled) duals of the standard model gauge group with extra vectorlike matter.

Another possibility, which is realized in the models discussed in Section 2., is that the scales of the Landau poles of the standard model gauge couplings are pushed up, beyond the GUT, string, or Planck scale. One way to realize this possibility is to construct $G_{S B}$ models with two scales, e.g. $M$ and $\Lambda$. In the models we will construct, $M$ will be a fundamental scale, which suppresses some nonrenormalizable operators, while $\Lambda$ is a dynamically generated scale. The ratio $\Lambda / M$ is a small parameter, which will generate the desired hierarchy of scales in the supersymmetry breaking sector. Although in these models $G_{S B}$ can have a rather large rank, causing multiple copies of messengers to appear, the dynamics may be such - due to the presence of the small parameter $\Lambda / M$ - that only a small number $(\leq 4$, in terms of $\mathbf{5}+\mathbf{5}^{*}$ of $\left.S U(5)\right)$ of these messengers is light, while the others are heavy, of order the GUT scale (or heavier). Thus the appearance of the Landau poles of the standard model gauge couplings is "postponed" until scales where a description in terms of a more fundamental (string) theory becomes valid.

\subsection{What is needed to build models with dynamical messengers?}

From the discussion in the previous sections, it is clear that a model with dynamical messenger fields has to incorporate the following ingredients. One needs, first of all, a supersymmetry-breaking theory, which has a large enough nonanomalous flavor symmetry (such that $G_{S M}$ can be embedded in it). In addition, the flavor group should be unbroken by the supersymmetry-breaking dynamics. Finally, in order for the Landau poles to be pushed up, we require that the dynamics is such that only a few (or none - see refs. [6], [7) light messenger fields appear in the spectrum. 


\section{Existence proof: models based on $\mathrm{SU}(\mathbf{N}) \times \mathrm{SU}(\mathbf{N}-\mathbf{2})$.}

In this section we will present the "existence proof" for such models. The model we construct is based on the $S U(N) \times S U(N-2)$ theories of supersymmetry breaking [8]. As we discuss later, the models are not phenomenologically acceptable as purely gauge-mediated models. However, they present the first example of models with dynamical messenger fields and by studying their dynamics in some detail, we will learn some general properties, which are expected to be shared by more realistic models.

\subsection{The tools: the $\mathrm{SU}(\mathrm{N}) \times \mathrm{SU}(\mathrm{M})$ theo- ries.}

As we discussed in Section 1.3, in order to construct models with dynamical messenger sectors, one requires a supersymmetry-breaking theory that has a large enough anomaly-free flavor symmetry (so that the standard model gauge group can be embedded in it). In addition, one would like to be able to establish that the flavor group is unbroken in the vacuum. At present, verifying this in the presence of supersymmetry breaking is only possible if the supersymmetry breaking dynamics is calculable, at least in the infrared.

We have studied in some detail the construction of supersymmetry-breaking theories based on supersymmetric QCD with gauged flavor symmetries. Following this "Rube Goldberg" (see A. Nelson's contribution) approach we arrive at a specific set of theories based on product gauge groups. The advantage of this approach is that models based on gauging flavor symmetries are relatively easy to analyze (since each of the gauge groups is supersymmetric QCD, the nonperturbative dynamics of which is well understood), yet the interplay of the gauge dynamics of the two groups gives rise to many new interesting nonperturbative phenomena, specific for the productgroup case [9].

By gauging the chiral flavor symmetries of supersymmetric QCD (and completing the representation to obtain the minimal anomaly-free content) we arrive at the following class of gauge theories: the gauge group is $S U(N) \times S U(M)$ with a matter content consisting of a single field, $Q$, that transforms as $(\square, \square)$ under the gauge group, $M$ fields, $\bar{L}$, transforming as $(\bar{\square}, \mathbf{1})$, and $N$ fields, $\bar{R}$, that transform as $(\mathbf{1}, \bar{\square})$. We note that these theories are chiral-no mass terms can be added for any of the matter fields. The detailed dynamics of these models was analyzed in refs. [9], [8]. Here we will only list the features relevant for model building.

We begin with the case $N=M$. The global vacuum in this case does not break supersymmetry - the theories in fact exhibit confinement without chiral symmetry breaking [8]. However, it is possible to show that there exist local minima which break supersymmetry and preserve a large enough subgroup of the flavor symmetries [7]. These local vacua (with $N=5$ ) have been recently used in the construction of some interesting models with dynamical messengers [7].

In the case $M=N-1$, it was shown in ref. [9] that with an appropriate superpotential added, the models break supersymmetry. In addition, the vacuum can occur in the calculable regime 11], and a large enough global symmetry $(S U(N-2))$ can be left unbroken. However, in these models, the global symmetry is anomalous, and gauging it requires adding "spectator" multiplets to obtain anomaly-free representations. The $S U(N) \times S U(N-1)$ models (with additional standard model representations) were used in ref. 12] to construct some models of dynamical supersymmetry breaking that unify the messenger and supersymmetry breaking sectors. The dynamics of these models is similar to the ones discussed below (for an important distinction, see comment at the end of Section 2.3).

The $M=N-2$ case was considered in ref. [8], [10. It was shown that upon adding an appropriate superpotential the models with odd $N$ break supersymmetry with a stable ground state and preserve a global, anomaly-free, symmetry $S P(N-3)$ (we use $S P(2)=S U(2)$ ). These models will comprise the supersymmetry breaking sector of the models we will construct below. The models with even $N$ have global supersymmetric vacua.

For completeness, we note that at present we do not know whether there exist stable 
supersymmetry-breaking vacua in the models with $M<N-2$. The main difficulty in establishing this lies in analyzing the classical flat directions.

\subsection{The $\mathrm{SU}(\mathrm{N}) \times \mathrm{SU}(\mathrm{N}-2)$ models: scales and messenger spectra.}

Given the results described above and the necessary conditions of Section 1.3, it is now straightforward to build phenomenological models with dynamical messengers. Since the $S U(N) \times$ $S U(N-2)$ models break supersymmetry and preserve an anomaly free global $S P(N-3)(N$-odd), one can identify a subgroup of the global symmetry with the standard model gauge group. The fields in the supersymmetry breaking sector then carry charges under both standard model and supersymmetry-breaking gauge groups, $G_{S M}$ and $G_{S B}=S U(N) \times S U(N-2)$. Embedding $G_{S M}$ into the $S P(N-3)$ global symmetry requires $N \geq 11$ (while consistency with grand unification is only possible for $N \geq 13$ ) [10].

We will not describe in detail the dynamics of these models here. We will only stress that the models realize the idea discussed in the previous section: the Landau poles for the standard model gauge groups are "postponed" until after the GUT (or string scale). Two scales are essential for the dynamics of these models. One scale, $M$, is the scale that suppresses the nonrenormalizable term in the superpotential, which is needed to lift the flat directions. The second scale - the strong coupling scale of the $S U(N)$ gauge group, $\Lambda, \Lambda \ll M$-is dynamically generated. Instead of using $M, \Lambda$ as the two scales, it is convenient to use $M, v$, where $v$ is the scale of some vacuum expectation value and is a function of both $M$ and $\Lambda$ [10. We will see below that the presence of two scales, $M$ and $v$, with a small ratio $v / M \ll 1$, generates the desired hierarchy of mass scales.

The dynamics of the models is such that at the scale $v(v \ll M)$ the $S U(N-2)$ gauge group is totally broken. Below the scale of $S U(N-2)$ breaking, the dynamics is governed by a pure $S U(N)$ gauge theory, with a dilaton and a number of moduli fields. Under the $S P(N-3)$ flavor group, the light moduli fields transform as two fundamentals and three singlets. At the scale

$\Lambda_{L}=v\left(\frac{v}{M}\right)^{\frac{N-5}{3}}$

the pure $S U(N)$ gauge theory confines and the dynamics below this scale is governed by the moduli and dilaton only. Moreover - and this is an important feature of these models pointed out by Shirman 11 —at energy scales below $\Lambda_{L}$, the dynamics of the moduli and dilaton fields is described by a calculable nonlinear supersymmetric sigma model. This fact allows us to compute the spectrum of the light excitations and confirm that the flavor symmetry is unbroken in the supersymmetry-breaking ground state; for details, see [10]. We note that the dynamics of this model is not entirely calculable - it is only the spectrum of the excitations lighter than $\Lambda_{L}$ that is calculable; the spectrum of strongly coupled bound states of gauginos and gauge bosons (of mass $\sim \Lambda_{L}$ ) of the pure $S U(N)$ gauge theory is beyond theoretical control. In this sense the model differs from the known calculable models of dynamical supersymmetry breaking, such as the "3-2" model.

As we already mentioned, the light spectrum only involves two fundamentals of the $S P(N-3)$ flavor group. Thus, gauging the standard model gauge group (say, in a way consistent with grand unification, requiring thus $N \geq 13$ ), the spectrum of light messengers consists of two pairs of $\mathbf{5}+\mathbf{5}^{*}$ representations of $S U(5)$. The masses of these light messengers are of order

$m=v\left(\frac{v}{M}\right)^{N-5}$.

Finally, the supersymmetry breaking scale is of order $F=m v$, i.e.

$\sqrt{F}=v\left(\frac{v}{M}\right)^{\frac{N-5}{2}}$.

The masses of all heavy messenger fields are of order $v$. Thus, they do not contribute to the running of the standard model gauge couplings below that scale. The Landau poles of the standard model are therefore "postponed" until scales higher than $v$. Beyond that scale, a more fundamental theory is expected to furnish the correct description of the dynamics. 


\subsection{Communication of supersymmetry breaking: the messenger $\mathrm{StrM}^{2}$ and the soft parameters.}

The communication of supersymmetry breaking is expected to work as in the usual models of gauge mediation - since the messenger fields are also a part of the supersymmetry breaking sector, they "learn" about supersymmetry breaking at tree level. The superpartners of the quarks, leptons, and standard model gauge bosons acquire supersymmetry-breaking masses at loop level. Thus, one expects that the order of magnitude of the soft masses is $\sim g^{2} m / 16 \pi^{2}$, as in the MGM models. We note that there are contributions to the supersymmetry-breaking soft masses of the standard model superpartners at two scales: both the heavy (of mass $v$ ) and light (of mass $m$ ) messengers contribute to the soft parameters (the contributions of the heavy and light messengers are of the same order of magnitude; for a discussion of the contribution of the heavy messenger vector superfields see ref. [15]).

The spectrum of light messengers in these models, however, differs in one crucial respect from the messenger spectrum of the MGM models. Since the dynamics of the supersymmetrybreaking-cum-messenger sector is governed by a complicated nonlinear sigma model, the masses of the messenger quarks and leptons receive contributions from both the superpotential and Kähler potential. The nontrivial Kähler potential contributions lead to a nonvanishing supertrace of the light messenger mass matrix. In fact, it is expected that in any model where the messengers participate in the breaking of supersymmetry, and the low energy dynamics can be described by a weakly coupled nonlinear sigma model, Str $M_{\text {mess }}^{2} \neq 0$. This can be seen by considering the tree level supertrace mass squared sum rule [13] for a general nonlinear sigma model:

Str $M^{2}=-2 R_{i j^{*}} K^{i l^{*}} K^{m j^{*}} W_{m} W_{l^{*}}^{*}$.

Here we use the notations of ref. [13]: $W_{m}$ is the gradient of the superpotential, $K^{i j^{*}}$ is the inverse Kähler metric, and $R_{i j^{*}}$ is the Ricci tensor of the Kähler manifold. In eq. (2.6) the trace is taken over all states in the sigma model (including the messenger singlets), however - and explicit examples confirm this - one does not expect a restriction of the supertrace on the space of states charged under a global symmetry to vanish for a Kähler manifold with nonzero curvature.

As a result of the nonvanishing supertrace, the light messengers' contribution to the soft scalar masses turns out to be logarithmically divergent; see ref. [14]. The divergent piece is:

$\delta_{L} m^{2} \sim-\frac{g^{4}}{256 \pi^{4}} \operatorname{Str} M_{m e s s}^{2} \log \frac{\Lambda^{2}}{m^{2}}$.

Here $\Lambda$ is the ultraviolet cutoff and $m$ is the scale of the light messengers (2.4), introduced earlier. The logarithm is cut off by the contributions of the heavy messenger fields; see discussion in ref. [14.

The explicit calculation of the light messenger spectrum in [10] shows that in the considered vacuum, $\operatorname{Str} M_{\text {mess }}^{2}>0$. Then eq. 2.7 implies that the scalar soft masses in this vacuum receive a large logarithmically enhanced negative contribution. Thus, the vacuum studied in ref. 10 can only be phenomenologically relevant if there are additional positive contributions to the soft scalar masses that offset the log-enhanced negative contribution due to the positive supertrace. A detailed numerical analysis shows that the positive contribution of the heavy messengers is not sufficient to cancel the large negative logarithmic contribution. Thus, the models can only be viable if in addition to the gauge mediation effects, there are also positive supergravity contributions to the soft scalar masses. In fact, upon examining the energy scales in these models, with $M=M_{\text {Planck }}=2 \times 10^{18} \mathrm{GeV}$, we find that for $N=13-17$, the scale $v=10^{16} \mathrm{GeV}$, while the supersymmetry breaking scale (2.5) is $\sqrt{F}=10^{10}$ $\mathrm{GeV}$ (the scale of the light messenger fields (2.4) is of order $m=10^{4} \mathrm{GeV}$ ). Thus, supergravity contributions are of the same order as the gaugemediated contributions. Therefore we refer to these models as "hybrid" models of supersymmetry breaking. We note that the scalar soft masses in the hybrid models receive contributions at three different energy scales - the (uncalculable) supergravity contribution at $M=M_{\text {Planck }}$ and the contributions from the heavy and light messengers at the scales $v$ and $m$, respectively. 
On the other hand, gauginos only receive contributions from gauge mediation, at the scales $v$ and $m$. One expects that in the "hybrid" models the renormalization group running will be different than that in pure supergravity or gauge mediated models - in particular, since only the squarks receive the negative supertrace contribution, it might be possible to obtain soft-parameter spectra with squarks and sleptons lighter than the gauginos.

Finally, we would like to stress that the scalar potential of the nonlinear sigma model that describes the infrared dynamics of the $S U(N) \times$ $S U(N-2)$ models is very complicated - we only studied the simplest solution - and it is not excluded that there exist vacua where the supertrace has a negative sign. We also note that a negative supertrace can not occur in the models 12 based on $S U(N) \times S U(N-1)$. This is essentially due to the fact that, as we mentioned in Section 2.1, the global $S U(N-2)$ symmetry is anomalous. 't Hooft anomaly matching then implies the existence of a massless fermion in the supersymmetry breaking sector, leading to a positive supertrace of the light messengers. The addition of extra matter to form anomaly-free representations can give a Dirac mass to this fermion 12, which is, however, a small higher-order effect and can not change the sign of the supertrace.

If models with dynamical messengers - or new vacua of the existing models - are found, with negative messenger supertrace, one expects to obtain a soft spectrum different than the one in the MGM models, with the scalar soft masses logarithmically enhanced with respect to the gaugino masses (we note that some models that have negative supertrace were constructed in refs. [16], 17). The phenomenological viability of such models deserves a closer investigation.

\section{What have we learned?}

In the course of this investigation, we showed that models with dynamical messengers can be constructed. We outlined the necessary requirements to build successful such models, and proposed a solution to the Landau pole problem, where the Landau poles are "postponed" to higher energy scales - beyond the GUT or string scales - where a more fundamental description of the dynamics is expected to take over.

We constructed an explicit example of such models, based on the $S U(N) \times S U(N-2)$ theories of dynamical supersymmetry breaking. We showed that the supersymmetry breaking dynamics leaves a large enough global symmetry unbroken, and calculated the detailed spectrum of light messenger fields.

We showed that, quite generally, in models with dynamical messenger fields, where the low-energy dynamics is described by a weekly coupled nonlinear sigma model, one expects the supertrace of the light messenger fields to be nonvanishing. We showed that this supertrace leads to logarithmically enhanced contributions to the soft scalar masses. Positivity of the soft scalar mass squared requires generally that the sign of the supertrace of the light messengers is negative.

Clearly, a lot of work remains to be done to find the "Standard Model" of gauge-mediated supersymmetry breaking (for some interesting new developments, see the contribution of $\mathrm{H}$. Murayama to this volume and refs. [6], [7]). As we previously emphasized, although the models constructed here are not acceptable as purely gauge-mediated models, but can only work as "hybrid" models, the detailed investigation of the supersymmetry-breaking dynamics and generation of the soft masses teaches us some general properties of such models. The lessons learned are likely to be useful in building models that are more aesthetically appealing and phenomenologically viable.

\section{REFERENCES}

1. M. Dine, A.E. Nelson, and Y. Shirman, hepph/9408384, Phys. Rev. D51 (1995) 1362.

2. N. Arkani-Hamed, C.D. Carone, L.J. Hall, and H. Murayama, hep-ph/9607298, Phys. Rev. D54 (1996) 7032;

3. I. Dasgupta, B.A. Dobrescu, and L. Randall, hep-ph/9607487, Nucl. Phys. B483 (1997) 95.

4. M. Dine and A.E. Nelson, hep-ph/9303230, Phys. Rev. D48 (1993) 1277. 
5. N. Seiberg, hep-th/9411149, Nucl. Phys. B435 (1995) 129.

6. H. Murayama, hep-ph/9705271, Phys. Rev. Lett. 79 (1997) 18.

7. S. Dimopoulos, G. Dvali, R. Rattazzi, and G.F. Giudice, hep-ph/9705307.

8. E. Poppitz, Y. Shadmi, and S.P. Trivedi, hepth/9606184, Phys. Lett. B388 (1996) 561.

9. E. Poppitz, Y. Shadmi, and S.P. Trivedi, hepth/9605113, Nucl. Phys. B480 (1996) 125.

10. E. Poppitz and S.P. Trivedi, hep-ph/9609529, Phys. Rev. D55 (1997) 5508.

11. Y. Shirman, hep-th/9608147, Phys. Lett. B389 (1996) 287.

12. N. Arkani-Hamed, J. March-Russell, and H. Murayama, hep-ph/9701286.

13. J. Wess and J. Bagger, Supersymmetry and Supergravity, Princeton UP, (1992).

14. E. Poppitz and S.P. Trivedi, hep-ph/9703246, Phys. Lett. B401 (1997) 38.

15. G.F. Giudice and R. Rattazzi, hep-ph/9706540.

16. L. Randall, hep-ph/9612426, Nucl. Phys. B495 (1997) 37.

17. B. Dobrescu, hep-ph/9703390, Phys. Lett. B403 (1997) 285. 